\title{
Repacking on Demand for Hierarchical Cellular Networks
}

\author{
HSIEN-MING TSAI \\ Senior Specialist, Research Institute, Quanta Computer Inc., Taoyuan, Taiwan, ROC \\ AI-CHUN PANG \\ Asst. Professor, Graduate Institute of Networking and Multimedia, Department of Computer Science and Information Engineering, \\ National Taiwan University, Taipei, Taiwan, ROC \\ YUNG-CHUN LIN \\ Ph.D. Candidate, Department of Computer Science and Information Engineering, National Chiao Tung University, Hsinchu, Taiwan, ROC \\ YI-BING LIN* \\ Chair Professor, Department of Computer Science and Information Engineering, National Chiao Tung University, Hsinchu, Taiwan, ROC
}

\begin{abstract}
In mobile telecommunications operation, radio channels are scarce resources and should be carefully assigned. One possibility is to deploy the hierarchical cellular network $(\mathrm{HCN})$. This paper studies a $\mathrm{HCN}$ channel assignment scheme called repacking on demand (RoD). RoD was originally proposed for wireless local loop networks. We expend this work to accommodate mobile HCN. A simulation model is proposed to study the performance of $\mathrm{HCN}$ with RoD and some previously proposed schemes. Our study quantitatively indicates that RoD may significantly outperform the previous proposed schemes.
\end{abstract}

Keywords: channel assignment, channel repacking, hierarchical cellular network, repacking on demand

\section{Introduction}

One of the most important issues in cellular network operation is capacity planning. Especially when the number of cellular subscribers grows rapidly, it is required that the cellular service provider increases its network capacity effectively. One possible solution is to deploy the hierarchical cellular network (HCN) $[5,11,13]$. As shown in figure 1 , the $\mathrm{HCN}$ consists of two types of base stations (BSs): micro BSs and macro BSs. A micro BS with low power transceivers provides small radio coverage (referred to as microcell), and a macro BS with high power transceivers provides large radio coverage (referred to as macrocell). The microcells cover mobile subscribers (MSs) in heavy teletraffic areas. A macrocell is overlaid with several microcells to cover all MSs in these microcells.

In a cellular network, radio channels must be carefully assigned to reduce the numbers of new call blockings as well as handoff call force-terminations. Several channel planning and assignment approaches have been proposed for HCN [1$4,7,14-18,20]$. Some studies focused on channel assignment according to the received radio signal strength $[3,15]$. Other studies $[2,4,7,20]$ investigated radio channel packing issues for channel reuse. A basic scheme called no repacking (NR) was described in [16]. In this scheme, when a call attempt (either a new call or a handoff call) for an MS arrives, the HCN first tries to allocate a channel in the microcell of the MS. If no idle channel is available in this microcell, the call attempt overflows

\footnotetext{
*Corresponding author.

E-mail: liny@csie.nctu.edu.tw
}

to the corresponding macrocell. If the macrocell has no idle channel, the call attempt is rejected. Call blockings and forceterminations of NR can be reduced by repacking techniques described as follows [21]. Consider a call attempt for a microcell $\mathrm{BS}_{i}$ that has no idle channel. In NR, this call attempt is served by the corresponding macrocell. If radio channels are available in $\mathrm{BS}_{i}$ later, this call can be transferred from the macrocell to $\mathrm{BS}_{i}$ again. The process of switching a call from the macrocell to the microcell is called repacking. Repacking increases the number of idle channels in a macrocell so that more macrocell channels can be shared by the call attempts where no channels are available in the microcells. Depending on the time when repacking is exercised, several schemes have been proposed. In always repacking (AR) $[1,14,17]$, the HCN always moves a call from the macrocell to the corresponding microcell as soon as a channel is released at that microcell. Some schemes $[8,19]$ perform repacking based on the moving speeds of MSs. In [8], the calls of slow-speed MSs are always moved from the macrocells to the corresponding microcells when these MSs move across the borders of microcells.

In $[6,19]$, repacking on demand (RoD) schemes were proposed. Unlike AR, RoD does not immediately perform repacking when a channel in a microcell is released. Instead, repacking is exercised only when it is necessary. Based on the speeds of MSs, the study in [19] investigated RoD for Exponential cell residence times and call holding times. The study in our previous work [6] investigated $\mathrm{RoD}$ for wireless local loop. This paper extends the work in [6] to accommodate mobile networks. We consider general distributions for both the cell residence times and call holding times. 


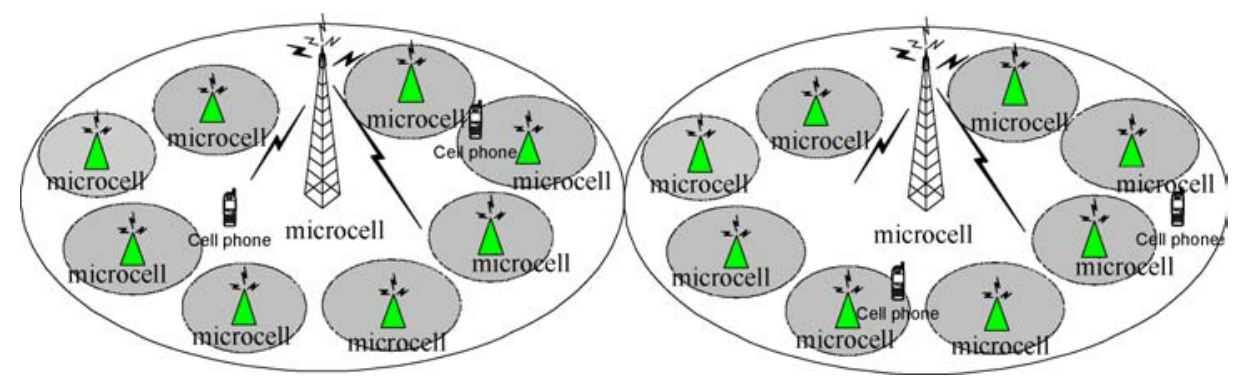

Figure 1. Hierarchical cellular network architecture.

Our study develops a simulation model to investigate the performance (i.e., the call blocking, force-termination, and incompletion) for NR, AR, and RoD. In Sections 2 and 3, we describe RoD. In Section 5.1, we propose a discrete event simulation model for HCN channel assignment. In Section 4, we use simulation experiments to compare NR, AR, and RoD. Our study quantitatively shows that RoD outperforms NR and AR.

\section{Repacking on demand for hierarchical cellular network (HCN)}

This section describes the RoD channel assignment and repacking procedures for $\mathrm{HCN}$. As shown in figure 2, when a call attempt is newly generated from or handed off to the $i$ th microcell, the HCN first tries to assign a channel in the $i$ th microcell to the call attempt (see figure 2(1) and (2)). If no idle channel is available in the $i$ th microcell, the call attempt overflows to the $j$ th macrocell that overlays with the $i$ th microcell. If the macrocell has idle channels, the HCN assigns one to the call attempt (figure 2(3) and (4)). Otherwise, RoD is exercised to identify repacking candidates (figure 2(5)). Every repacking candidate is an ongoing call that satisfies the following criteria:

Criterion 1. The call occupies a channel in the $j$ th macrocell. Criterion 2. The microcell of this call (i.e., the microcell where the call party resides) has an idle channel.

RoD selects one repacking candidate to hand off from the macrocell to the microcell where the call resides (see figure 2(6) and (7)). Then the reclaimed macrocell channel is used to serve the call attempt (figure 2(8)). If RoD cannot find any repacking candidate, the call attempt is rejected; i.e., the new call is blocked or the handoff call is force-terminated (figure 2(9)).

We propose two policies to handle the repacking candidates in RoD at Step 6 in figure 2. Random RoD (RoD-R) randomly selects a repacking candidate with the same probability. Load Balancing RoD (RoD-L) selects the repacking candidate whose microcell has the least traffic load. Both RoD$\mathrm{R}$ and RoD-L can be adopted by an HCN that utilizes radio systems such as GSM/PCS1900 [13] or WCDMA [5], where the handoff decision is made by the network.

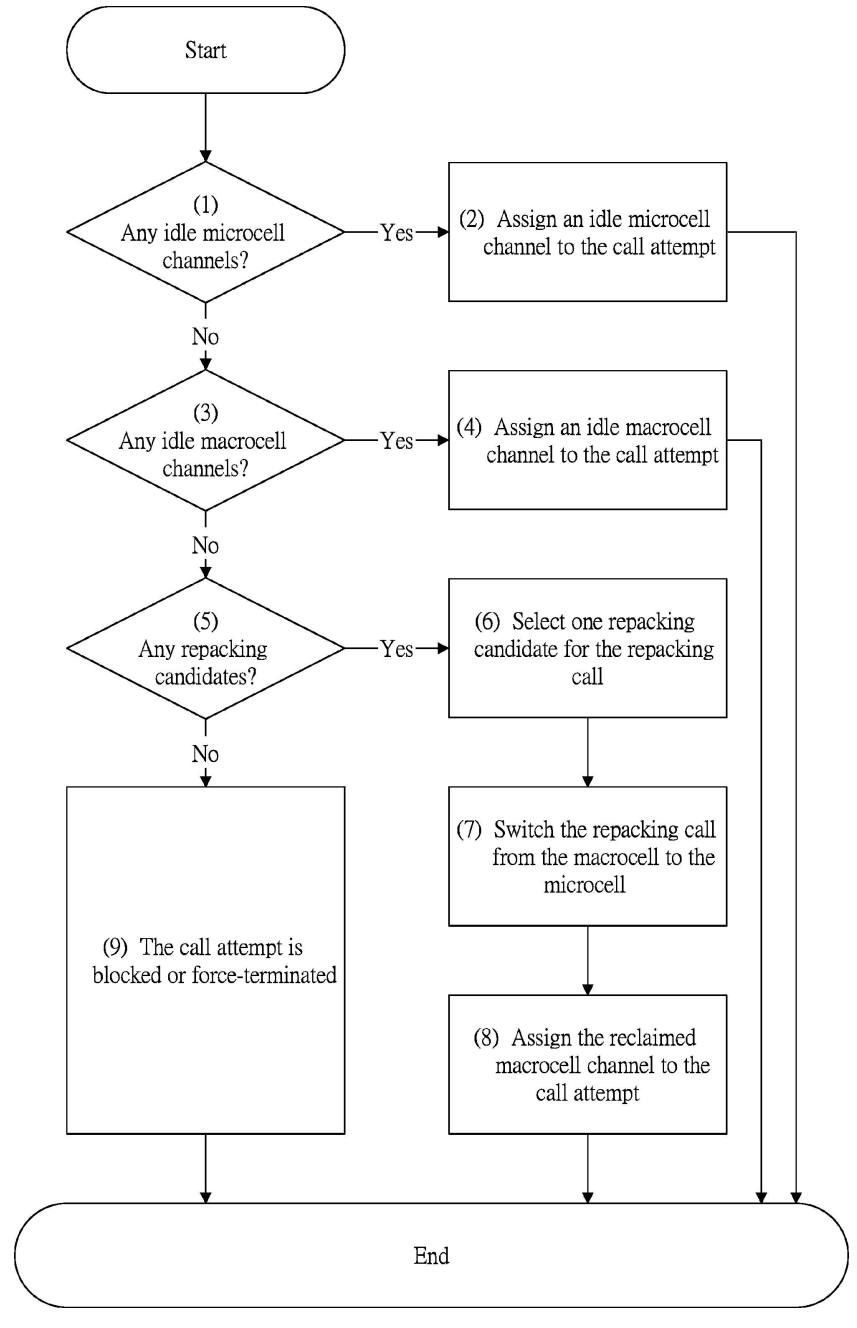

Figure 2. Channel assignment with RoD.

\section{System model for $\mathrm{HCN}$}

This section describes the input parameters and output measures for the HCN system model. Our model can accommodate any cell configurations. For the demonstration purpose, we consider a wrapped mesh cell configuration as shown in figure 3. This configuration consists of four macrocells. Each macrocell covers $4 \times 4$ microcells. The wrapped topology simulates unbounded $\mathrm{HCN}$ so that the boundary cell effects can be ignored [12]. Without loss of generality, the MS moves to 


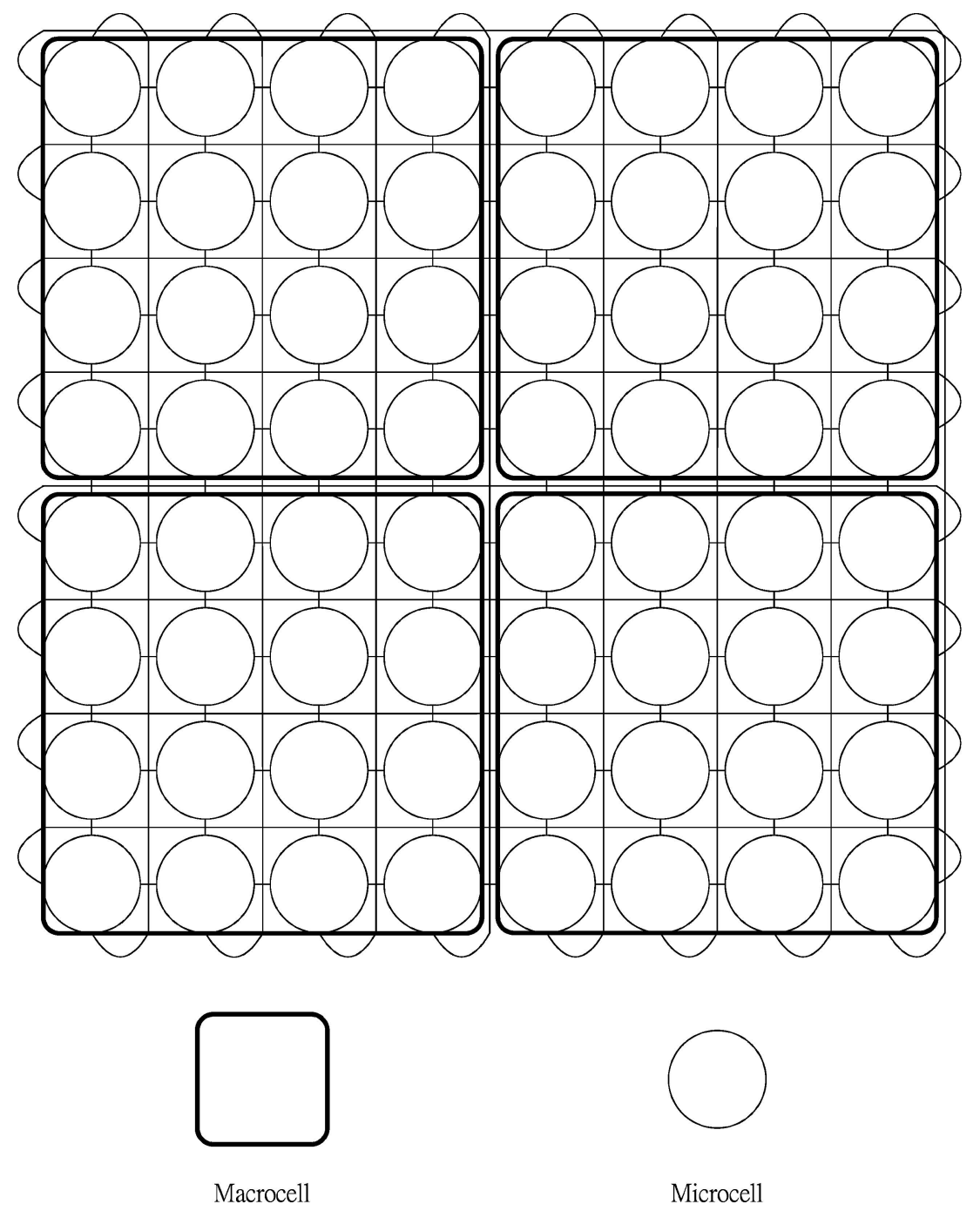

Figure 3. Hierarchical cellular network with wrapped mesh configuration.

one of the four neighbor microcells with the same probability (i.e., 0.25). Three types of input parameters are considered in our model.

System Parameters: Each macrocell has $C$ radio channels, and each microcell has $c$ radio channels.

Traffic Parameters: The call arrivals to a microcell (for both incoming and outgoing calls) form a Poisson stream with rate $\lambda$. The expected call holding time is $1 / \mu$.

Mobility Parameters: The expected microcell residence time of an MS is $1 / \eta$.

Several output measures are defined in this study:

$P_{b}$ : the blocking probability that a new call is blocked

$P_{f}$ : the force-termination probability that a successfully connected call is force-terminated because of handoff failure

$P_{n c}$ : the incomplete probability that a new call is blocked or a connected call is force-terminated

$H$ : the expected number of handoffs occurred during a call
Based on the source and the target cells of handoff, figure 4 shows five types of handoffs, and five handoff measures are defined.

$H_{m m}$ : the expected number of handoffs from a microcell to another microcell during a call (figure 4(a))

$H_{m M}$ : the expected number of handoffs from a microcell to a macrocell during a call (figure 4(b))

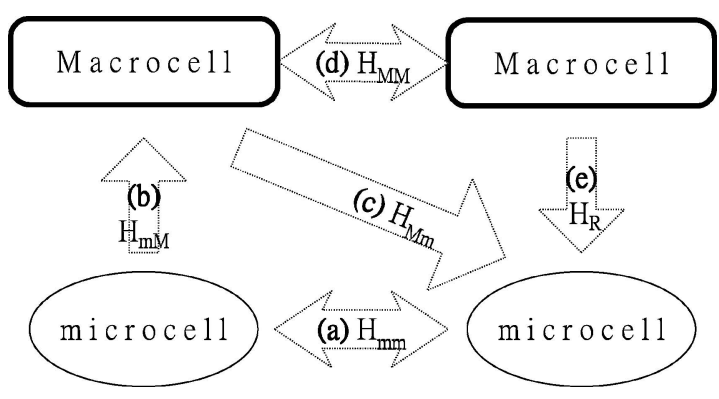

Figure 4. Handoff types. 
$H_{M m}$ : the expected number of handoffs from a macrocell to a microcell during a call (figure 4(c))

$H_{M M}$ : the expected number of handoffs from a macrocell to another macrocell during a call (figure 4(d))

$H_{R}$ : the expected number of repackings during a call (figure 4(e))

From the above description, $H$ can be expressed as

$$
H=H_{m m}+H_{m M}+H_{M m}+H_{M M}+H_{R} .
$$

Table 1 lists the notation described in this section, which will be used in the remainder of the paper.

When the MS residence times are exponentially distributed, RoD may be model by multi-dimensional Markov chain. Since the details are tedious and the assumption is quite artificial, we will not work on this approach. Instead, we use simulation approach to model more realistic scenarios with general MS residence time distributions. In Appendix A, we describe a

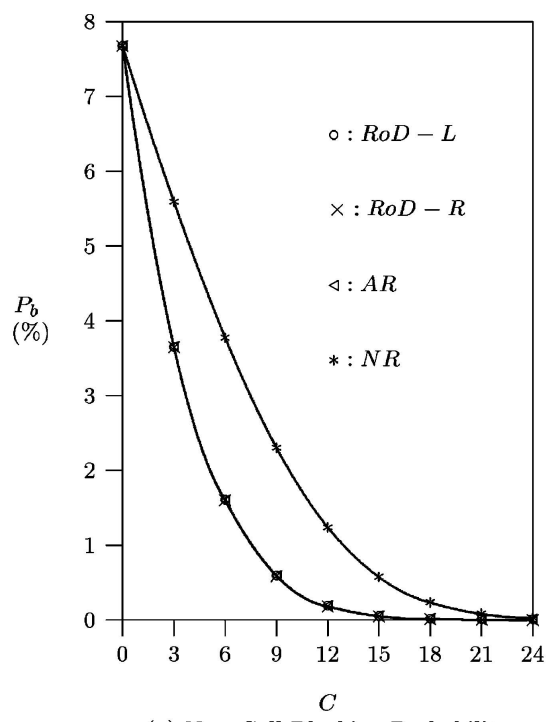

(a) New Call Blocking Probability

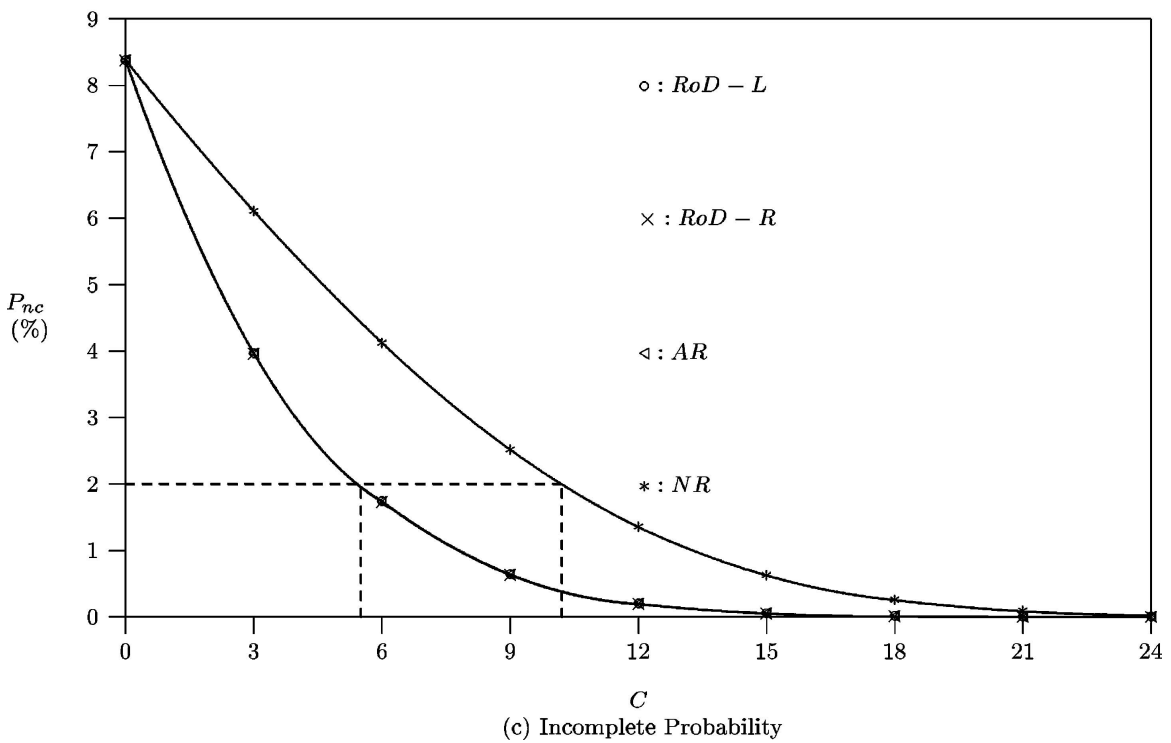

(c) Incomplete Probability

Figure 5. Effects of $C$ on $P_{b}, P_{f}$ and $P_{n c}\left(c=10, \lambda=7 \mu, V_{\mu}=1 / \mu^{2}, \eta=0.1 \mu\right.$ and $\left.V_{\eta}=1 / \eta^{2}\right)$.

Figure 5. Efrects of $C$ on $P_{b}, P_{f}$ and $P_{n c}\left(c=10, \lambda=7 \mu, V_{\mu}=1 / \mu^{2}, \eta=0.1 \mu\right.$ discrete simulation model for RoD. This simulation model is partially validated by several analytic models $[6,13]$, and the details are omitted.

\section{Results and discussions}

Based on the simulation model developed in Appendix A, we compare NR, AR, RoD-R and RoD-L in terms of the output measures defined in Section 3. In our numerical examples, the radio channel number is $c=10$ for every microcell. We assume that the call holding times have a Gamma distribution with mean $1 / \mu$ and variance $V_{\mu}$ (the typical value for $1 / \mu$ is 1 minute). We also assume that the microcell residence times have a Gamma distribution with mean $1 / \eta$ and variance $V_{\eta}$. The Gamma distribution is considered because it can approximate many distributions as well as measured data (see Lemma 3.9 in [9]). Note that when $V_{\mu}=1 / \mu^{2}\left(V_{\eta}=1 / \eta^{2}\right)$,

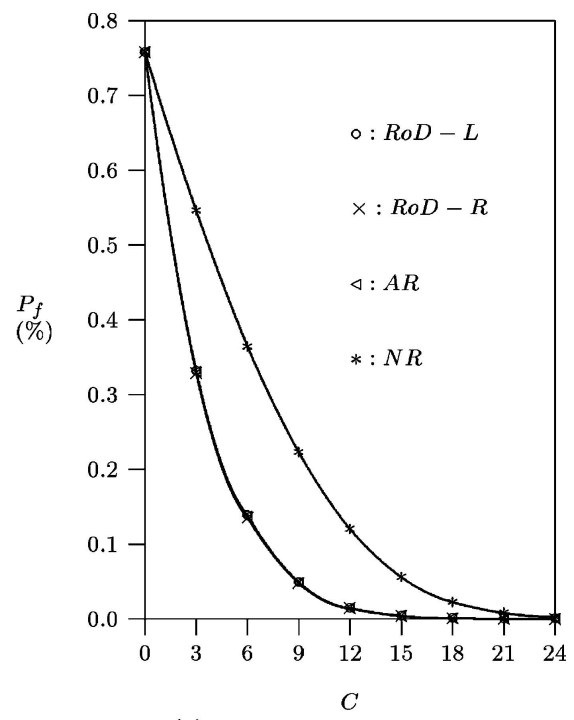

(b) Force-Terminaton Probability 


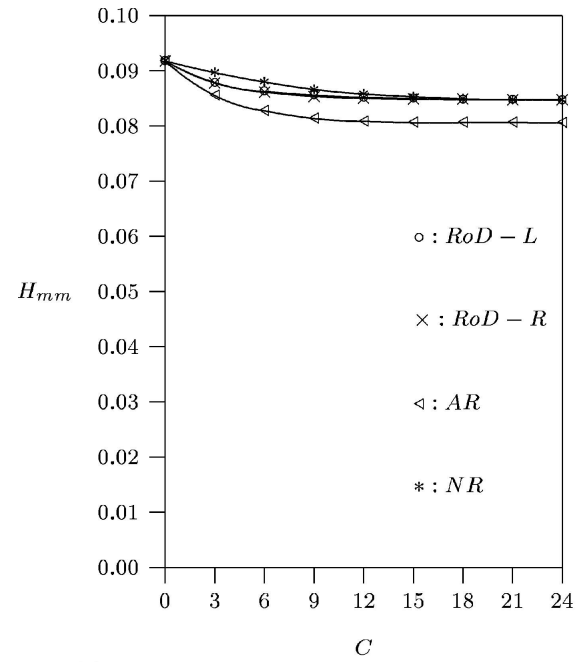

(a) Expected Number of Microcell to Microcell Handoffs

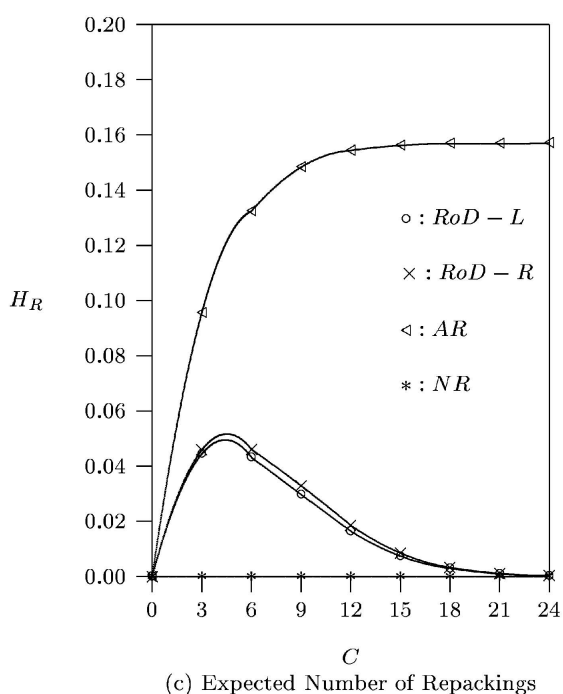

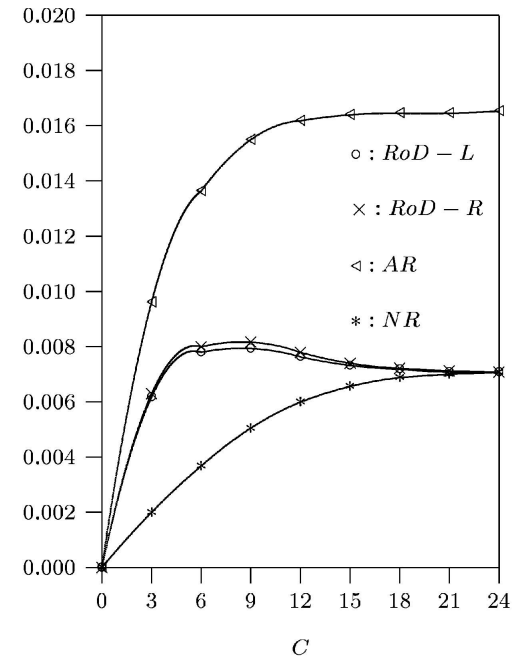

(b) Expected Number of Microcell to Macrocell Handoffs

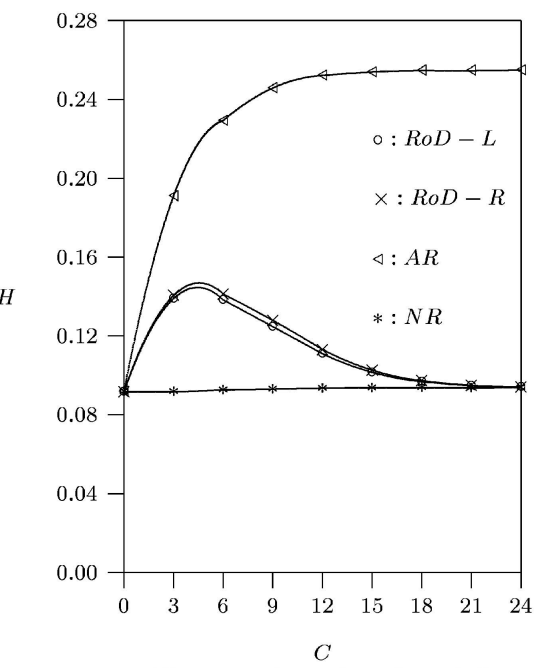

(d) Expected Number of Handoffs

Figure 6. Effects of $C$ on $H_{m m}, H_{m M}, H_{R}$ and $H\left(c=10, \lambda=7 \mu, V_{\mu}=1 / \mu^{2}, \eta=0.1 \mu\right.$ and $\left.V_{\eta}=1 / \eta^{2}\right)$.

the Gamma distribution becomes an Exponential distribution. The call arrivals to every microcell form a Poisson process with the arrival rate $\lambda$. In each simulation run, $10^{5}-10^{6}$ call arrival events per microcell are executed to ensure that simulation results are stable. Furthermore, we run replications such that the confidence intervals of the $99 \%$ confidence levels are within $3 \%$ of the mean values in most cases. The effects of the input parameters are described as follows.

Effect of the macrocell channel number $C$. Figures 5(a), (b) and (c) plot $P_{b}, P_{f}$ and $P_{n c}$ as functions of $C$, where $\lambda=7 \mu, V_{\mu}=1 / \mu^{2}, \eta=0.1 \mu$ and $V_{\eta}=1 / \eta^{2}$. These figures show the intuitive results that for all approaches under investigation, $P_{b}, P_{f}$ and $P_{n c}$ decrease as $C$ increases. When $C$ is small, macrocell channels are the bottleneck resources. Increasing $C$ significantly improves the $P_{b}, P_{f}$ and $P_{n c}$ performance. When $C$ is larger than a threshold $C^{*}$, the macrocell channels are no longer the bottleneck, and adding extra macrocell channels only insignificantly reduces $P_{b}, P_{f}$ and $P_{n c}$. The importance of our study is that for any specific input parameters, we can find the threshold $C^{*}$. For example, $C^{*}=12$ for RoD and AR in figure 5 . Figures 6 (a)-(d) plot $H_{m m}, H_{m M}, H_{R}$ and $H$ as functions of $C$, where $\lambda=7 \mu, V_{\mu}=1 / \mu^{2}, \eta=0.1 \mu$ and $V_{\eta}=1 / \eta^{2}$. For all approaches, figure 6(a) shows that $H_{m m}$ is a decreasing function of $C$. On the other hand, $H_{m M}$ (figure 6(b)) is an increasing function of $C$. These phenomena are due

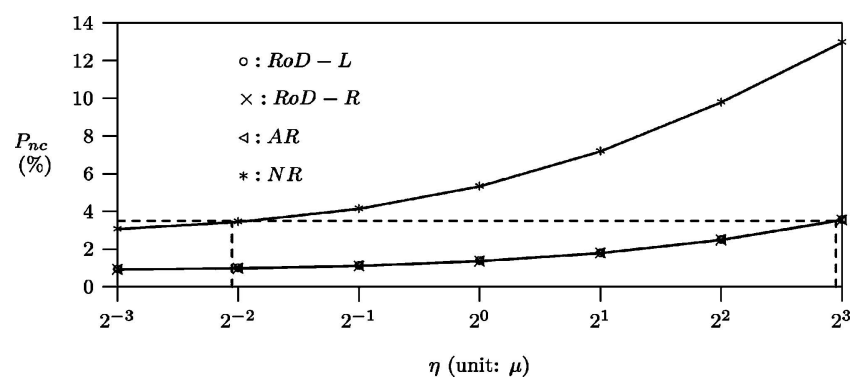

Figure 7. Effects of $\eta$ on $P_{n c}\left(C=8, c=10, \lambda=7 \mu, V_{\mu}=1 / \mu^{2}\right.$ and $V_{\eta}=$ $\left.1 / \eta^{2}\right)$. 


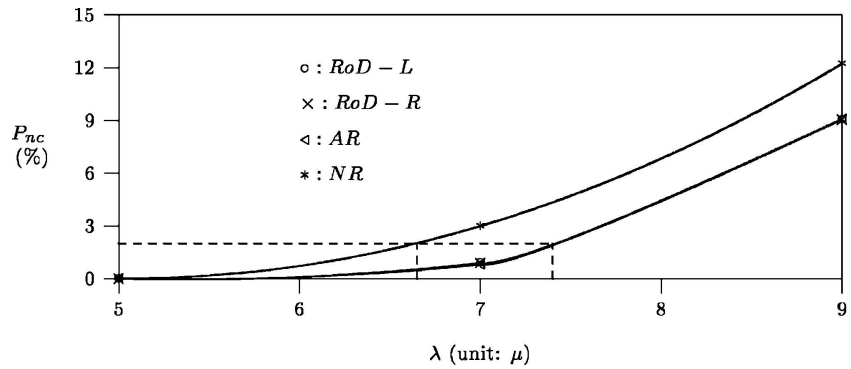

Figure 8. Effects of $\lambda$ on $P_{n c}\left(C=8, c=10, V_{\mu}=1 / \mu^{2}, \eta=0.1 \mu\right.$ and $\left.V_{\eta}=1 / \eta^{2}\right)$.

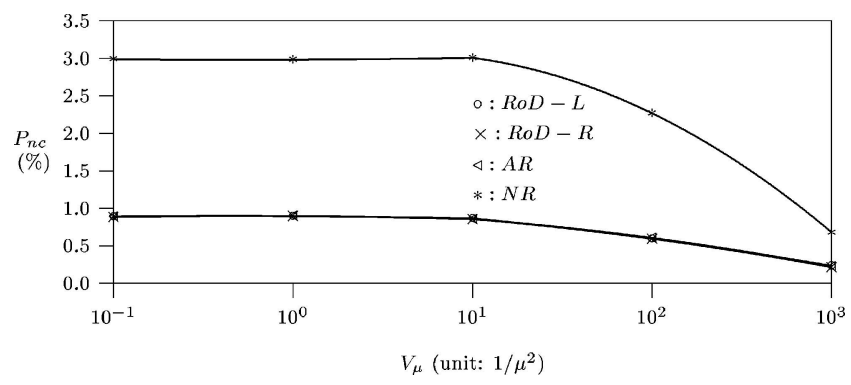

Figure 9. Effects of variance $\left(V_{\mu}\right)$ of call holding time on $P_{n c}(C=8, c=10$, $\lambda=7 \mu, \eta=0.1 \mu$ and $\left.V_{\eta}=1 / \eta^{2}\right)$.

to the fact that when $C$ increases, all handoff activities involving macrocell channels will increase, and the number of pure microcell to microcell handoffs will decrease. The performance figures for $H_{M m}$ and $H_{M M}$ are similar to that for $H_{m M}$, and the details are omitted. Figure 6(c) shows that when $\mathrm{AR}$ is exercised, $H_{R}$ increases as $C$ increases for the following reason. Increasing $C$ results in more repacking candidates, and more repackings are exercised. On the other hand, for RoD-R and RoD-L, $H_{R}$ increases and then decreases as $C$ increases. This non-trivial phenomenon is
Table 1

Notation.

\begin{tabular}{|c|c|}
\hline Notation & Description \\
\hline & System parameters \\
\hline$C$ & the number of radio channels in a macrocell \\
\hline$c$ & the number of radio channels in a microcell \\
\hline & Traffic parameters \\
\hline$\lambda$ & the call arrival rate to a microcell \\
\hline $1 / \mu$ & the expected call holding time \\
\hline & Mobility parameters \\
\hline $1 / \eta$ & the expected microcell residence time \\
\hline & Output measures \\
\hline$P_{b}$ & the blocking probability \\
\hline$P_{f}$ & the force-termination probability \\
\hline$P_{n c}$ & the incomplete probability \\
\hline$H_{m m}$ & the expected number of microcell-to-microcell handoffs per call \\
\hline$H_{m M}$ & the expected number of microcell-to-macrocell handoffs per call \\
\hline$H_{M m}$ & the expected number of macrocell-to-microcell handoffs per call \\
\hline$H_{M M}$ & the expected number of macrocell-to-macrocell handoffs per call \\
\hline$H_{R}$ & the expected number of repackings per call \\
\hline$H$ & the expected number of handoffs per call \\
\hline
\end{tabular}

explained as follows. When $C$ is small, macrocell channels are the bottleneck resources. Increasing $C$ results in more repacking candidates, and more on-demand repackings are exercised (just like AR). When $C$ is large $(C>5$ in figure $6(\mathrm{c}))$, macrocell channels are no longer the bottleneck. Increasing $C$ results in fewer blockings as well as force-terminations, and less on-demand repackings are needed. Therefore $H_{R}$ decreases as $C$ increases. Figure 6(d) shows the net effects of repackings and all types of handoffs. In this figure, $H$ is an increasing function for NR and AR. For RoD-R and RoD-L, as $C$ increases, $H$ increases and then decreases.

Comparison of NR, AR, RoD-R and RoD-L. Figures 5(a), (b) and (c) indicate that repacking approaches (AR, RoD-R and RoD-L) have the same $P_{b}, P_{f}$ and $P_{n c}$ values, which

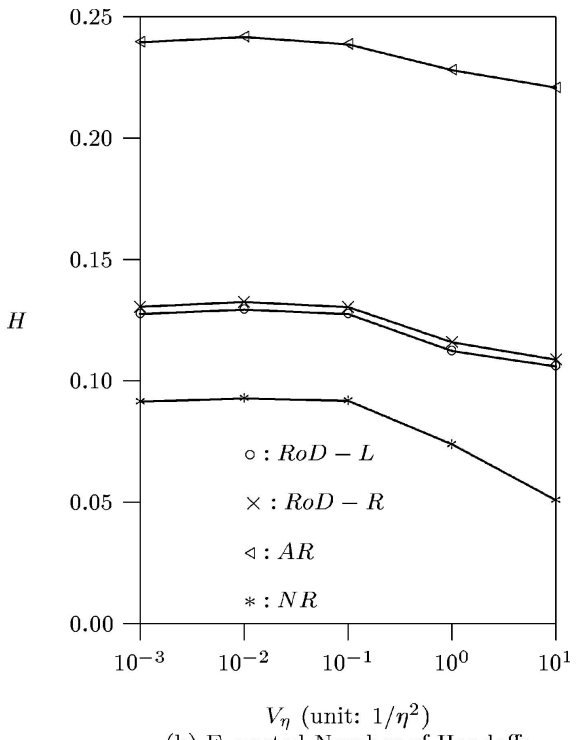

(b) Expected Number of Handoffs

Figure 10. Effects of variance $\left(V_{\eta}\right)$ of microcell residence time on $P_{n c}$ and $H\left(C=8, c=10, \lambda=7 \mu, V_{\mu}=1 / \mu^{2}\right.$ and $\left.\eta=0.1 \mu\right)$. 
are much less than those for NR. If the $\mathrm{HCN}$ is engineered at $P_{n c}=2 \%$ (see the horizontal dashed line in figure 5(c)), $C=5.5$ for repacking approaches and $C=10.5$ for NR, and repacking approaches can save 5 macrocell channels over NR.

Figure 6(c) shows that $H_{R, A R} \gg H_{R, R o D-R}>H_{R, R o D-L}$ $>H_{R, N R}=0$. This result is the same as that for WLL, and the details will not be given. The reader is referred to our previous work for the details [6]. Figure 6(d) indicates that $H_{A R} \gg H_{R o D-R}>H_{R o D-L}>H_{N R}$. Specifically, when the $\mathrm{HCN}$ is engineered at $C=5.5$ (i.e., $P_{n c}=2 \%$ ) RoD-R and RoD-L reduce $62 \%$ and $66 \%$ of handoffs over AR, respectively.

Effect of the Mean Microcell Residence Time $1 / \eta$. Figure 7 plots $P_{n c}$ as a function of $\eta$, where $C=8, \lambda=7 \mu, V_{\mu}=$ $1 / \mu^{2}$, and $V_{\eta}=1 / \eta^{2}$. This figure shows an intuitive result that for all approaches, $P_{n c}$ increases as $\eta$ increases. The non-trivial result is that to keep the same $P_{n c}$ performance (e.g., $P_{n c}=3.5 \%$ ), the repacking approaches can support much faster MSs ( $2^{5}$ times the $\eta$ value) than NR does.

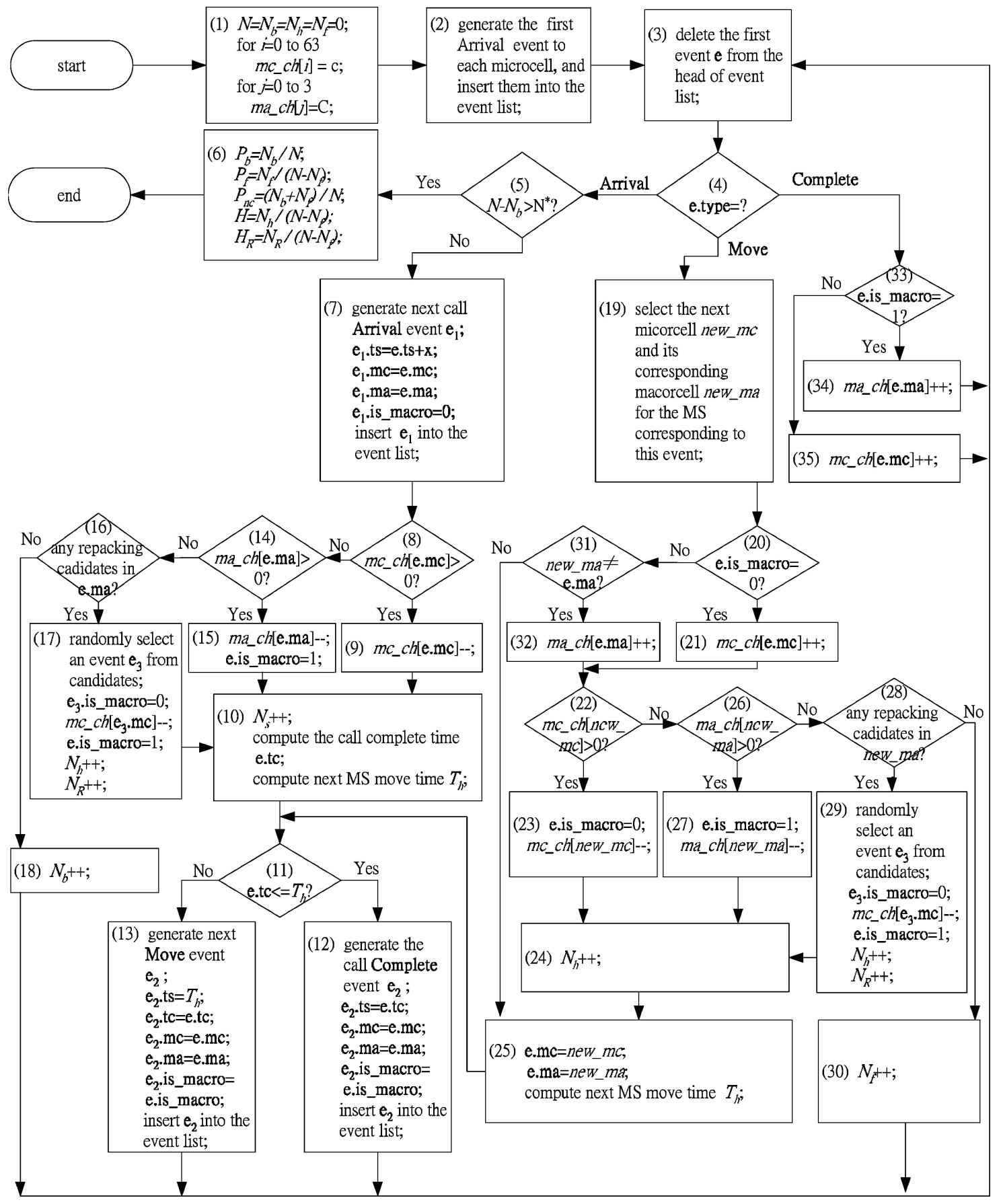

Figure 11. Simulation flow chart for RoD-R. 
Effect of the Arrival Rate $\lambda$. Figure 8 plots $P_{n c}$ as an increasing function of $\lambda$, where $C=8, V_{\mu}=1 / \mu^{2}, \eta=0.1 \mu$ and $V_{\eta}=1 / \eta^{2}$. It shows that to keep the same $P_{n c}$ performance (e.g., $P_{n c}=2 \%$ ), repacking approaches can support more call arrivals (more than $11 \%$ ) than NR.

Effect of the Variance $V_{\mu}$ for the Call Holding Time. Figure 9 plots $P_{n c}$ as a decreasing function of variance $V_{\mu}$, where $C=8, \lambda=7 \mu, \eta=0.1 \mu$ and $V_{\eta}=1 / \eta^{2}$. Note that, for the call holding time distributions with the same mean value $1 / \mu$, the standard deviation $\sigma=\sqrt{V_{\mu}}$ By the Chebyshev's Inequality, the probability that the call holding times are out of range $\left[1 / \mu-\frac{5 \sqrt{V_{\mu}}}{3}, 1 / \mu+\frac{5 \sqrt{V_{\mu}}}{3}\right]$ is smaller than 36 percent for all $V_{\mu}$ values. For example, if $V_{\mu}=100 / \mu^{2}$, then $\frac{5 \sqrt{V_{\mu}}}{3}=50 / 3 \mu$ and the probability that the call holding time exceeds $53 / 3 \mu$ is smaller than 36 percent. We note that as $V_{\mu}$ increases, more large and small call holding times are observed. More short call holding times implies that more calls are completed before next new call attempts arrive or next handoff attempts are exercised. Thus the numbers of blocked calls and force-terminated calls decrease.

Effect of the Variance $V_{\eta}$ for the Microcell Residence Time. Figures 10(a) and (b) plot $P_{n c}$ and $H$ as functions of variance $V_{\eta}$, where $C=8, \lambda=7 \mu, V_{\mu}=1 / \mu^{2}$ and $\eta=0.1 \mu$. These figures show that for all approaches, $P_{n c}$ and $H$ decrease as $V_{\eta}$ increases. From the residual life theorem [10], the mean value of the first microcell residence time increases as $V_{\eta}$ increases, which implies that more calls will complete in the first microcell before they are handed off to the next cells. Therefore, both $P_{n c}$ and $H$ drop as $V_{\eta}$ increases.

\section{Conclusions}

This paper studied repacking on Demand (RoD) for channel assignment in a hierarchical cellular network (HCN). We developed simulation models to investigate the RoD performance on the blocking probability $P_{b}$, the force-termination probability $P_{f}$, the incomplete probability $P_{n c}$ and the expected number of handoffs $H$ during a call. We compared RoD with other HCN channel assignment schemes including no repacking (NR) and always repacking (AR). We showed that RoD and AR have the same $P_{b}, P_{f}$ and $P_{n c}$ performance. Compared with NR, both RoD and AR reduce the $P_{b}, P_{f}$, and $P_{n c}$ performance at cost of increasing the number of handoffs. With the same $P_{n c}$ performance, the repacking approaches can support much faster MSs or more call arrivals than NR does. Moreover, our study indicated that RoD results in much less handoffs than AR does.

\subsection{Simulation model}

This appendix describes the discrete event simulation model for RoD-R. Other strategies (such as NR, AR and RoD-L) can be studied by similar simulation models, and the details are omitted. In this simulation model, three types of events are defined to represent call arrival, call completion, and MS movement. These events are inserted into the event list, and are deleted/processed from the event list in the non-decreasing timestamp order. A simulation clock is maintained to indicate the simulation progress, which is the timestamp of the event being processed. The following attributes are defined for an event e:

- The type attribute indicates the event type. An Arrival event represents a new call arrival. A Move event represents an MS movement from one cell to another. A Complete event represents a call completion.

- The ts attribute indicates the time when the event occurs.

- The tc attribute indicates the time when the call corresponding to e will complete. Note that tc $\geq$ ts.

- The mc attribute indicates the microcell where the MS (corresponding to this event) resides.

- The ma attribute indicates the macrocell where the MS (corresponding to this event) resides.

- The is_macro attribute indicates whether the call corresponding to this event occupies a macrocell channel. If a macrocell channel is occupied, is_macro $=1$. Otherwise, is_macro $=0$.

An array $m c_{-} c h[i]$ is used to represent the number of the idle channels of microcell $i$. Another array $m a_{-} c h[j]$ is used to represent the number of the idle channels of macrocell $j$. When a handoff occurs, two variables new $m c$ and new_ma are used to indicate the next micro and macro cells where the MS moves into. The outputs measured in the simulation are the number $N$ of total call arrivals during the simulation, the number $N_{b}$ of blocked calls, the number $N_{f}$ of force-terminated calls, the number $N_{h}$ of handoffs. From the above output measures, we can compute

$$
\begin{aligned}
P_{b} & =\frac{N_{b}}{N}, \quad P_{f}=\frac{N_{f}}{N-N_{b}}, \quad P_{n c}=\frac{N_{b}+N_{f}}{N}, \\
H_{R} & =\frac{N_{R}}{N-N_{b}} \quad \text { and } \quad H=\frac{N_{h}}{N-N_{b}} .
\end{aligned}
$$

Figure 11 shows the simulation flow chart for RoD-R, which is described as follows. Step 1 initializes the input parameters. Step 2 generates the first Arrival event for each microcell and inserts these events into the event list. In Steps 3 and 4, the next event $\mathbf{e}$ in the event list is processed based on its type. There are three cases:

Case I. e.type $=$ Arrival: At Step 5, if $N-N_{b}>N^{*}$ (in our simulation, $\left.N^{*}=6 \times 10^{5} \times 64\right)$, then Step 6 computes the output measures using (2), and the simulation terminates. Otherwise, Step 7 generates the next Arrival event $\mathbf{e}_{\mathbf{1}}$ for the same microcell (i.e., $\mathbf{e}_{\mathbf{1}} \cdot \mathbf{m c}=\mathbf{e} . \mathbf{m c}, \mathbf{e}_{\mathbf{1}} \cdot \mathbf{m a}=\mathbf{e} . \mathbf{m a}$ and $\mathbf{e}_{\mathbf{1}}$.is_macro=0). The timestamp of $\mathbf{e}_{\mathbf{1}}$ equals e.ts plus the inter call arrival time generated by a random number generator. Then $\mathbf{e}_{\mathbf{1}}$ is inserted into the event list. At Step 8, if microcell e.mc has idle channels (i.e., $m c_{-} c h[\mathbf{e} . \mathbf{m c}]>0$ ), then a channel is assigned to the incoming call. Then $m c_{-} c h[\mathbf{e} . \mathbf{m c}]$ is decremented by 1 at Step 9. Step 10 computes the call 
completion time e.tc as e.ts plus the call holding time. Step 10 also determines the MS move time $T_{h}$ when the MS moves out of the microcell. $T_{h}$ equals e.ts plus the cell residence time. Then Step 11 determines the next event (i.e., a Move event or Complete event) for the call corresponding to e. If the MS will move to another cell after call completion (i.e., e.tc $\leq T_{h}$ ), then Step 12 is executed to generate a Complete event $\mathbf{e}_{2}$ where $\mathbf{e}_{2} . \mathbf{t s}=\mathbf{e} . \mathbf{t c}, \mathbf{e}_{2} . \mathbf{m c}=\mathbf{e} . \mathbf{m c}$, $\mathbf{e}_{2} \cdot \mathbf{m a}=\mathbf{e} . \mathbf{m a}$ and $\mathbf{e}_{2}$.is_macro $=$ e.is_macro. Event $\mathbf{e}_{2}$ is inserted into the event list. On the other hand, if the MS moves to another cell before the call completion (i.e., e.tc $>T_{h}$ ) at Step 11, then Step 13 is executed to generate the next Move event $\mathbf{e}_{2}$ with the timestamp $T_{h}$ for this call (i.e., $\mathbf{e}_{2} . \mathbf{t s}=T_{h}, \mathbf{e}_{2} . \mathbf{t c}=\mathbf{e . t c}, \mathbf{e}_{2} \cdot \mathbf{m c}=\mathbf{e} \cdot \mathbf{m c}, \mathbf{e}_{2} \cdot \mathbf{m a}=$ e.ma and $\mathbf{e}_{2}$.is_macro $=$ e.is_macro). Event $\mathbf{e}_{2}$ is inserted into the event list.

At Step 8, if microcell e.mc has no idle channel (i.e., $m c \_c h[\mathbf{e} . \mathbf{m c}]=0$ ), the call attempt overflows to macrocell e.ma. Step 14 checks if macrocell e.ma has idle channels (i.e., $m a_{-} c h[$ e.ma] $>0$ ). If so, a macrocell channel is assigned to the incoming call at Step 15. In this case, $m a_{-} c h[\mathbf{e} . \mathbf{m a}]$ is decremented by 1 , and e.is_macro $=1$. The simulation proceeds to execute Steps 10-12 (or 13).

If macrocell e.ma has no idle channel (i.e., $\left.m a_{-} c h[\mathbf{e} . \mathbf{m a}]=0\right)$ at Step 14 , then Step 16 checks if there are repacking candidates in macrocell e.ma. If so, Step 17 randomly selects a repacking candidate (i.e., a call corresponding to a Complete or Move event $\mathbf{e}_{3}$ ) to be handed off from macrocell e.ma to microcell $\mathbf{e}_{3} . \mathbf{m c}$ (i.e., $\mathbf{e}_{3}$.is_macro is set to 0 ), and the reclaimed macrocell channel is assigned to the incoming call (i.e., e.is_macro is set to 1$)$. Step 17 also decrements $m c_{-} c h\left[\mathbf{e}_{3} . \mathbf{m c}\right]$ by 1 , and increments both $N_{R}$ and $N_{h}$ by 1 . Then the simulation proceeds to execute Steps 10-12 (or 13). If no repacking candidate is found at Step 16, the incoming call is blocked, and $N_{b}$ is incremented by 1 at Step 18 .

Case II. e.type $=$ Move: Step 19 selects the next microcell $n e w \_m c$ and its macrocell new $m a$ for the MS corresponding to event e. At Step 20, if the call of the MS occupies a microcell channel (i.e., e.is_macro $=0$ ), the occupied channel of microcell e.mc is released $\left(m c_{-} c h[\mathbf{e} . \mathbf{m c}]\right.$ is incremented by 1 at Step 21). At Step 22, if microcell $n e w \_m c$ has idle channels (i.e., $m c \_c h\left[n e w \_m c\right]>0$ ), a channel is assigned to the handoff call. Then at Step 23, the call is handed off to the microcell (i.e., e.is_macro $=0$ ), and $m c_{-} c h\left[n e w \_m c\right]$ is decremented by 1 . Step 24 increments $N_{h}$ by 1 . Step 25 updates the current cell for the call (i.e., e.mc $=n e w \_m c$ and e.ma $=n e w \_m a$ ) and computes the next MS move time $T_{h}$ for the call. Then the simulation proceeds to execute Steps 11 and 12 (or 13).

At Step 22, if all channels in the new microcell are busy (i.e., $m c_{-} c h\left[n e w \_m c\right]=0$ ), then Step 26 checks whether macrocell new ma has an idle channel. If so (i.e., $\left.m a_{-} c h\left[n e w \_m a\right]>0\right)$, a macrocell channel is assigned to the handoff call. Then at Step 27, the call is handed off to the macrocell (i.e., e.is_macro is set to 1), and $m a_{-} c h\left[n e w \_m a\right]$ is decremented by 1 . The simulation proceeds to execute Steps 24, 25, 11 and 12 (or 13). On the other hand, if no channel is available in macrocell new_ma at Step 26, then Step 28 checks if there are repacking candidates in new_ma. If so, Step 29 is executed to repack the calls (same as Step 17), and the simulation proceeds to execute Steps 24, 25, 11 and 12 (or 13). At Step 28, if no repacking candidate is found, the call is force-terminated and Step 30 is executed to increment $N_{f}$ by 1 .

At Step 20, if the call occupies a macrocell channel, then Step 31 checks if the MS is moving out of its macrocell e.ma. If so (i.e., new $\_m a \neq \mathbf{e} . \mathbf{m a}$ ), the call is handed off to the new cell. Step 32 increments $m a_{-}$ch [e.ma] by 1; i.e., the occupied channel of e.ma is released. Then the simulation proceeds to execute Steps 22-30 or to execute Steps 22-25, 11 and 12 (or 13). At Step 31, if the MS does not move out of its macrocell (i.e., e.ma $=$ new_ma), the simulation proceeds to execute Step 25 and then Steps 11 and 12 (or 13).

Case III. e.type $=$ Complete: At Step 33, if the call occupies a macrocell channel (i.e., e.is_macro $=1$ ), then Step 34 is executed to increment $m a_{-} c h$ [e.ma] by 1 . Otherwise, Step 35 is executed to increment $m c_{\_} c h$ [e.mc] by 1 .

To accommodate RoD-L, we only need to modify Steps 17 and 29 of the flowchart in figure 11. The simulation model is partially validated by the analytic model in [6] for the no mobility case. The details are omitted.

\section{Acknowledgment}

We would like to thank the anonymous reviewers. Their comments have significantly improved the quality of this paper. This work was sponsored in part by NSC Excellence project NSC93-2752-E-0090005-PAE, NSC 93-2213-E009-100, NTP VoIP Project under grant number NSC 92-2219-E-009-032, IIS/Academia Sinica, ITRI/NCTU Joint Research Center, Quanta, National Science Council under contract NSC 92-2213-E-002-049, CCL/ITRI under contract T1-92021-6, Microsoft and Intel.

\section{References}

[1] R. Beraldi, S. Marano and C. Mastroianni, A reversible heirarchical scheme for microcellular systems with overlaying macrocells, in: Proc. of IEEE Infocom (1996) pp. 51-58.

[2] L.J. Cimini, G.J. Foschini, C.-L.I and Z. Miljanic, Call blocking performance of distributed algorithms for dynamic channel allocation in microcells, IEEE Tran. on Comm. 42(8) (1994) 2600-2607.

[3] B.O.P. Gudmundson, (Sollentuna, SE), H. Eriksson, (Vallentuna, SE) and O.E. Grumlund, (Kista, SE), Method of effecting handover in a mobile multilayer cellular radio system. U.S. Patent (1995).

[4] C.-J. Ho, C.-T. Lea and G.L. Stuber, Call admission control in the microcell/macrocell overlaying system. IEEE Tran. on Vehicular Tech. 50(4)(2001) 992-1003.

[5] H. Holma and A. Toskala, WCDMA for UMTS (John Wiley \& Sons, 2000).

[6] H.-N. Hung, Y.-B. Lin, N.-F. Peng and H.-M. Tsai, Repacking on demand in two-tier WLL. Accepted and to appear in IEEE Transactions on Wireless Communications. 
[7] C.-L. I, L.J. Greenstein and R.D. Gitlin, A microcell/macrocell cellular architecture for low- and high-mobility wireless users. IEEE JSAC 11(6) (1993) 885-891.

[8] B. Jabbari and W.F. Fuhrmann, Teletraffic modeling and analysis of flexible heirachical cellular networks with speed-sensitive handover strategy. IEEE JSAC 15(8) (1997) 1539-1548.

[9] F.P. Kelly, Reversibility and Stochastic Networks (John Wiley \& Sons Ltd., 1979).

[10] L. Kleinrock, Queueing Systems; Vol. I: Theory (Wiley, 1975).

[11] X. Lagrange, Multitier cell design, IEEE Communications Magazine 35(8) (1997) 60-64.

[12] Y.-B. Lin and V.W. Mak, Eliminating the boundary effect of a largescale personal communication service network simulation, ACM Tran. on Modeling and Computer Simulation 4(2) (1994) 165-190.

[13] Y.-B. Lin, W.-R. Lai and R.J. Chen, Performance analysis for dual band PCS networks. IEEE Tran. on Computers 49(2) (2000) 148-159.

[14] K. Maheshwari and A. Kumar, Performance analysis of microcellization for supporting two mobility classes in cellular wireless networks. IEEE Tran. on Vehicular Tech. 49(2) (2000) 321-333.

[15] P.A. Ramsdale, (Walden, GB) and P.S. Gaskell, (Shelford, GB), Handover techniques. U.S. Patent (1994).

[16] S.S. Rappaport and L.-R. Hu, Microcellular communication systems with hierarchical macrocell overlays: Traffic performance models and analysis, in: Proceedings of the IEEE 82(9) (1994) 1383-1397.

[17] R. Steele, M. Nofal and S. Eldolil, Adaptive algorithm for variable teletraffic demand in highway microcells. Electronics Letters 26(14) (1990) 988-990.

[18] F. Valois and V. Veque, Preemption policy for hierarchical cellular network. In: 5th IEEE Workshop on Mobile Multimedia Communication (1998) pp. 75-81.

[19] F. Valois and V. Veque, QoS-oriented channel assignment strategy for hierarchical cellular networks. IEEE PIMRC 2 (2000) 1599-1603.

[20] L.-C. Wang, G.L. Stuber and C.-T. Lea, Architecture design, frequency planning, and performance analysis for a microcel/macrocell overlaying system. IEEE Tran. on Vehicular Tech. 46(4) (1997) 836-848.

[21] P.A. Whiting and D.W. McMillan, Modeling for repacking in cellular radio, in: 7th U.K. Teletraffic Symp. (1990).

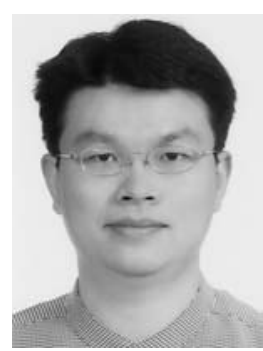

Hsien-Ming Tsai was born in Tainan, Taiwan, R.O.C., in 1973. He received the double B.S. degrees in Computer Science \& Information Engineering (CSIE) and Communication Engineering, the M.S. degree in CSIE, and the Ph.D. degree in CSIE from National Chiao-Tung University (NCTU), Taiwan, in 1996, 1997, and 2002, respectively. He is currently a research specialist in Quanta Research Institute, Quanta Computer Inc. His research interests are in the areas of cellular protocols
(UMTS/GPRS/GSM/DECT), cellular multimedia (MPEG-4 Audio/Speech), and embedded systems. He is an IEEE member.

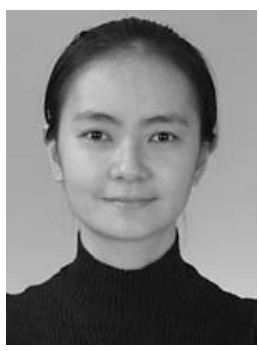

Ai-Chun Pang was born in Hsinchu, Taiwan, R.O.C., in 1973. She received the B.S., M.S. and Ph.D. degrees in Computer Science and Information Engineering from National Chiao Tung University (NCTU) in 1996, 1998 and 2002, respectively. She joined the Department of Computer Science and Information Engineering, National Taiwan University (NTU), Taipei, Taiwan, as an Assistant Professor in 2002. Her research interests include design and analysis of personal communications services network, mobile computing, voice over IP and performance modeling.

E-mail: acpang@csie.ntu.edu.tw

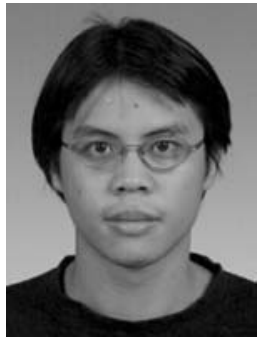

Yung-Chun Lin was born in Kaohsiung, Taiwan, R.O.C., in 1978. He received the B.S. and M.S. degrees in Computer Science and Information Engineering (CSIE) from National Chiao-Tung University (NCTU), Taiwan, in 2001, 2003, respectively. $\mathrm{He}$ is currently pursuing the Ph.D. degree in CSIE. His research interests include design and analysis of a personal communications services network, the cellular protocols (UMTS/GPRS/GSM), and mobile computing.

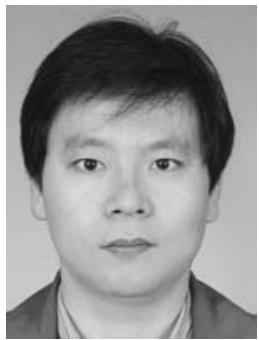

Yi-Bing Lin received his BSEE degree from National Cheng Kung University in 1983, and his Ph.D. degree in Computer Science from the University of Washington in 1990. From 1990 to 1995, he was with the Applied Research Area at Bell Communications Research (Bellcore), Morristown, NJ. In 1995, he was appointed as a professor of Department of Computer Science and Information Engineering (CSIE), National Chiao Tung University (NCTU). In 1996, he was appointed as Deputy Director of Microelectronics and Information Systems Research Center, NCTU. During 1997-1999, he was elected as Chairman of CSIE, NCTU. His current research interests include design and analysis of personal communications services network, mobile computing, distributed simulation, and performance modeling. Dr. Lin has published over 150 journal articles and more than 200 conference papers. Lin is an Adjunct Research Fellow of Academia Sinica, and is Chair Professor of Providence University. Lin serves as consultant of many telecommunications companies including FarEasTone and Chung Hwa Telecom. Lin is an IEEE Fellow and an ACM Fellow.

E-mail: liny@csie.nctu.edu.tw 